This item was submitted to Loughborough's Research Repository by the author.

Items in Figshare are protected by copyright, with all rights reserved, unless otherwise indicated.

\title{
Anharmonic acoustic effects during DNA hybridization on an electrochemical quartz crystal resonator
}

PLEASE CITE THE PUBLISHED VERSION

https://doi.org/10.1016/j.electacta.2018.03.014

PUBLISHER

(C) Elsevier

VERSION

AM (Accepted Manuscript)

\section{PUBLISHER STATEMENT}

This work is made available according to the conditions of the Creative Commons Attribution-NonCommercialNoDerivatives 4.0 International (CC BY-NC-ND 4.0) licence. Full details of this licence are available at: https://creativecommons.org/licenses/by-nc-nd/4.0/

\section{LICENCE}

CC BY-NC-ND 4.0

\section{REPOSITORY RECORD}

Swarbrick, Samantha, Igor Efimov, Victor P. Ostanin, David Klenerman, Alexandra Stolzing, and Sourav K. Ghosh. 2019. "Anharmonic Acoustic Effects During DNA Hybridization on an Electrochemical Quartz Crystal Resonator". figshare. https://hdl.handle.net/2134/32872. 


\section{Anharmonic acoustic effects during DNA hybridization on an electrochemical quartz crystal}

\section{resonator}

Samantha Swarbrick ${ }^{1}$, Igor Efimov ${ }^{1,2 *}$, Victor P. Ostanin ${ }^{3}$, David Klenerman ${ }^{3}$, Alexandra Stolzing ${ }^{1}$, Sourav K. Ghosh ${ }^{1 *}$

${ }^{1}$ Centre for Biological Engineering, Loughborough University, Holywell Park, LE11 3TU Leicestershire, UK

${ }^{2}$ Interdisciplinary Biomedical Research Centre, Nottingham Trent University, Clifton Lane, Nottingham, NG11 8NS, UK.

${ }^{3}$ Department of Chemistry, Cambridge University, Lensfield Road, Cambridge, CB2 1EW, UK

Keywords: electrochemical quartz crystal microbalance; acoustic detection of biomarkers; anharmonic oscillations; single-stranded DNA hybridization; electrochemical impedance 


\section{ABSTRACT}

The paper describes a sensor for single-stranded DNA (ssDNA) biomarker based on anharmonic acoustic signals arising during hybridization with complementary thiolated ssDNA functionalised on the gold electrode of an electrochemical quartz crystal resonator. The steps of sensor preparation and hybridization are carried out in an electrochemical microfluidic flowcell. While the electrochemical impedance spectroscopy does not allow a definitive interpretation, the changes in resonance frequency and third Fourier harmonic current of the resonator on actuation at the fundamental mode indicate formation of a flexibly bound layer. The functionalization and hybridization steps monitored by the anharmonic detection technique (ADT) are described with a simple model based on Duffing nonlinear equation.

\section{INTRODUCTION}

Detection of single-stranded DNA (ssDNA) is of wide interest in biomedical diagnostics and pharmaceutical manufacturing. Optical fluorescence is one of the most widely used methods in the detection of ssDNA. Although fluorescence-based methods have shown unprecedented sensitivity, the need for multiple reagents and steps has restricted these methods from use in the point-of-care or real-time setting $[1,2]$. In this paper, we investigate the feasibility of an electrochemical quartz crystal microbalance (EQCM) for direct and rapid detection of ssDNA using the nonlinear acoustic response of the quartz resonator. A sensor is developed by functionalizing thiolated ssDNA to one of the gold electrodes of the EQCM. This ssDNA has a complementary sequence to the target ssDNA in solution, and therefore acts as a specific 
biomolecular receptor for the latter. The binding event is recorded by electrochemical methods, particularly measuring changes of charge transfer resistance $R_{\mathrm{ct}}$ or double layer capacitance $C_{\mathrm{dl}}$, which are assessed in electrochemical impedance spectroscopy (EIS) [3, 4]. If the gold electrode is part of the EQCM, the change in resonance frequency indicates the hybridization event as a mass change in the simplest case [5, 6, 19-21].

In practice, these two methods EIS and EQCM have crucial disadvantages when they are applied to detection of DNA hybridization. In EIS, it is not clear how the charge transfer resistance should change when thiol-bound ssDNA is complemented by a matching strand. The interpretation of EQCM resonance frequency shift is also challenging as the frequency shift can either be negative or positive in the case of rigid or flexible binding respectively, depending on the dominance of mass or elastic loading. We observed that Thiolthiol-bound ssDNA under the influence of electrode potential resembles resembled the second case, i.e. where elastic loading is dominant over mass loading. As a result, the positive resonance frequency shifts reflects not a variation in mass but rather change in the spring constant of the bonds between the sensor and the attached analyte. Therefore, although the EQCM offers a direct and rapid platform for measurement, there is a need for an improved method for quantification of flexible adsorbate, such as ssDNA. An anharmonic detection technique, or ADT, has been reported recently to overcome the abovementioned issues and measure flexible adsorbates, such as microparticles, with a strong quantitative correlation and transduction specificity [7-10]. A QCM is largely linear at modest amplitudes, i.e. when driven by a harmonic (or purely sinusoidal) actuation near its fundamental resonance frequency, it responds predominantly at the same frequency. The response at higher odd Fourier harmonics is significantly low. It may be noted that even Fourier harmonics are not transduced in a thickness-shear mode quartz resonator. However, the 
interaction forces between the QCM oscillating in thickness shear mode and the surface-bound flexible adsorbate projected along the direction of the quartz oscillation (which is piezoelectrically transduced) are anharmonic (not harmonic) and distort the harmonic oscillation of the QCM. As a result, the binding of flexible adsorbates modifies the amplitude of higher odd Fourier harmonic due to piezoelectric transduction (even harmonics are not transduced in a thickness-shear mode quartz). In the anharmonic detection technique (ADT), we consider the change in amplitude in the third Fourier harmonic, which is measured at three times the drive frequency, as fifth or higher odd harmonics are considerably low in amplitude. It may be clarified here that although the frequency of the third Fourier harmonic; which is-(equal to three times the drive frequency,-) lies close to the third overtone resonance (as the drive frequency is Elose-set to the fundamental resonance), the two are different conceptually. While the third overtone refers to a higher mode of resonance of an oscillator, a third Fourier harmonic signal (i.e. three times the drive frequency) may be produced in principle even in a nonlinear oscillator with a single resonance mode -by driving at the with a-single (fundamental) mode of resonance-It may be noted, however, although such-that a single-mode a-quartz resonator is practically impossible. This is where ADT differs from an impedance analyzer or a ring-down analyzer, which measures the change in frequency at a chosen resonance mode by driving at or around that the same mode, whereas ADT measures the change in amplitude of a higher Fourier harmonic (usually third, which is near the third overtone resonance) by driving near the fundamental resonance frequency.

In this paper, we investigate by means of EIS and ADT, the steps of developing an ssDNA sensor (surface functionalisation) and the hybridization event (sensing). The functionalisation and hybridization steps are carried out in a microfluidic flowcell, and the measurements are 
taken in liquid after each step. The experimental results show satisfactory agreement with the theory, governed by the basic equations of a nonlinear QCM response with flexibly-bound adsorbate. In particular, the third Fourier harmonic signal obtained from the experiment exactly fit to a cubic expression as predicted by the Duffing equation and as elaborated in the paper. The cubic shape holds true both for clean QCM and for QCM with bound analyte, with the only difference being in the value of the pre-exponent.

\subsection{Modelling the anharmonic influence of non-rigid load}

In an earlier paper [10], we treated the problem of anharmonic influence of non-rigid load in its general case by considering a damped driven oscillator. In this paper, we have neglected losses in the theoretical model, and therefore omitted the drive force and treated the problem as free oscillation. This simplified the analytical expression of the result without any considerable impact on the agreement with the experimental results as the change in dissipation due to biomolecular binding at any stage is negligible. The resistance (real part of acoustic impedance) measured at any step in our experiment (with or without DNA) remained the same, i.e. 220 Ohm, as obtained from Kanazawa equation.

Let us consider the model of a particle bound to the quartz resonator surface with a bond length of $l$ (Fig 1). As the frequency of oscillation of the resonator $(14.3 \mathrm{MHz}$ in our case) is significantly higher than the natural frequency of shear oscillation of the particle-bond system $\left(\omega_{\underline{s}}=\left(k / m_{s}\right)^{1 / 2}\right)$, the particle remains nearly at the same location. As a result, the bond stretches and changes angle, as shown in Fig 1.

The oscillations in the system are described by two coupled differential equations (Eq.1 and Eq. 2), with mutual motions of the particle and the quartz resonator both parallel to the quartz 
surface. We model quartz as a nonlinear oscillator using the one-dimensional Duffing equation, neglecting damping and external drive force as mentioned above.

$$
\ddot{x}+\omega_{0}^{2} x=-\beta x^{3}+\frac{N S}{M_{q}} F_{x}
$$

Here, $x$ - displacement of quartz, $N$ - number of particles attached per unit area, $S$ active area of

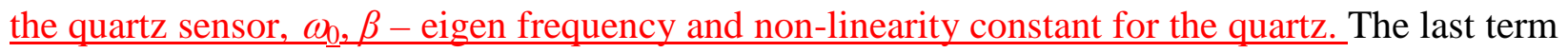
in Eq. 1 is the pulling force from NS adsorbed particles, where $N\left(1 / \mathrm{cm}^{2}\right)$ is surface density of particle coverage and $S\left(\mathrm{~cm}^{2}\right)$ is the total active area of the sensor, and $M_{q}$ is the nodal mass of the crystal, which is equal to half of the total mass [6]. Since the particle remains nearly stationary during the oscillation of the resonator, its motion is described by the following differential equation, where $F_{X}$ is the projection of the total particle-bond force on quartz plane.

$$
m_{s} \ddot{X}=-F_{x}=k\left(\sqrt{x^{2}+l^{2}}-l\right) \frac{x}{\sqrt{x^{2}+l^{2}}} \approx \frac{k x^{3}}{2 l^{2}} \text {, as } x<<l
$$

Here, $m_{\underline{s}}$ is the mass of attached particle, $X$ is the displacement of adsorbed particle placed at the origin and $k$ is force constant of attachment bond. As the particle remains stationary in the laboratory reference frame, the extension of the bond is given by $\left(\sqrt{x^{2}+l^{2}}-l\right)$, and $x$ / $\sqrt{x^{2}+l^{2}}$ is the factor that projects the elastic force $k\left(\sqrt{x^{2}+l^{2}}-l\right)$ (which is along the bond) onto the direction of oscillation.

Since the displacement of the resonator is small compared to the bond length, i.e. $x<<$, the anharmonicity in the spring constant of the bond can be neglected, and the bond can be treated as a linear spring as in Eq. 2. Other notations are: * displacement of quartz, $X$ displacement of adsorbed particle placed at the origin, $a$ - amplitude of oscillations in the quartz, $N$ number of 
particles attached, $S$ active area of the quartz, $k$ force constant of attachment bond, $\gamma$ cubic anharmonic force, $m_{s}$ - mass of attached particle, $\omega_{s}=\left(k / m_{s}\right)^{1 / 2}$ frequency of attachment bond, $t_{0,}, \beta$ eigen frequency and non-linearity constant for the quartz.

Substituting Eq.2 into Eq.1 results in the following equation for quartz oscillations:

$$
\ddot{x}+\omega_{0}^{2} x=-\beta x^{3}-\frac{N S}{M_{q}} \frac{k}{2 l^{2}} x^{3}
$$

The two terms on the right side of Eq.3 describe the motion of the harmonic oscillator in an anharmonic field of the fourth order.

$$
U_{4}=\frac{M_{q}}{4}\left(\beta+\frac{N S}{M_{q}} \frac{k}{2 l^{2}}\right) x^{4}
$$

The first term in parentheses is due to anharmonicity of the quartz itself, and the second is due to the particle-resonator interaction. Therefore harmonic but relatively long bonds generate anharmonic potential for oscillation of the quartz parallel to the surface.

Solution of Eq.3 is very well known (see for example references [11] or [12]). The fundamental resonance frequency shifts with higher oscillation amplitude due to drive-level dependence. For small oscillation amplitude $a$, the shift is proportional to square of the oscillation amplitude:

$$
\omega=\omega_{0}+\frac{3}{8 \omega_{0}}\left(\beta+\frac{N S}{M_{q}} \frac{k}{2 l^{2}}\right) a^{2}
$$

The first Fourier harmonic of the oscillator displacement is given by

$$
x^{(0)}=a \cos \omega t
$$


The third Fourier harmonic of the oscillator displacement is proportional to the third power of amplitude.

$$
x^{(3)}=\frac{a^{3}}{32 \omega_{0}^{2}}\left(\beta+\frac{N S}{M_{q}} \frac{k}{2 l^{2}}\right) \cos 3 \omega t
$$

Eq.5 and Eq.7 contain terms that depend on the concentration of adsorbed particles. This can be used for their quantitative detection. Positive (anti-Sauerbrey) frequency shift, described by Eq.5, was observed experimentally in the case of attachment of microbeads and bacteria [13-18]. Moreover, from Eq.5 and Eq.7, a simple relationship follows between the shifts in resonance frequency and the third Fourier harmonic amplitude due to binding of particles with respect to some reference state at a given actuation amplitude $a$.

$$
\frac{\omega-\omega_{0}}{\omega_{0}}=\frac{3}{8} \frac{a^{2} \beta}{\omega_{0}^{2}} \frac{x^{(3)}-x_{0}^{(3)}}{x_{0}^{(3)}}
$$

It is interesting to note that although Eq.8 contains only parameters of the quartz and drive amplitude, and no characteristics of the bound layer, it relates the shifts in fundamental resonance frequency $\omega_{0}$, and the third Fourier harmonic amplitude $x^{(3)}$ for each step of functionalization and binding.

As clarified earlier in the 'Introduction', the model describes the change in the amplitude of the third Fourier harmonic due to nonlinear effects when the quartz oscillator is driven near its fundamental resonance, and has no direct causative relation to the existence of the third overtone resonance.

\section{EXPERIMENTAL}

2.1 ADT instrument design 
The-A dedicated electronic instrument, which may be described as a "Nonlinear Network Analyzer”, has been designed, buildt and used for our experiments_10]. The basic principle of the instrument schematic along with a diagram was published in one of our earlier works [8]. The instrument is capable of driving a large variety of piezoelectric oscillators at a range of amplitude (0 up to 40V) at Radio Frequencies (RF $100 \mathrm{kHz}$ to 300MHz), and recording complex (real and imaginary) electrical signal (current and voltage) at three frequencies synchronously and sensitively (noise $\sim 1 \mu \mathrm{V} / \mathrm{Hz}^{1 / 2}$ ) using 12 heterodyne receivers. In our experiments, we recorded complex current and voltage signals at the drive frequency (first Fourier harmonic 1f) and three times the drive frequency (third Fourier harmonic 3f). The piezoelectric quartz oscillator acts as an actuator and a microphone at the same time. The driving and pickup electrodes are the same, i.e. only two electrodes used, as in a Quartz Crystal Microbalance (QCM). The microvolt-level third harmonic signals were separated from the powerful drive signal applied at the fundamental resonance by appropriate highly linear passive filtering network. For convenience, the experimental data will be reported in terms of normalized or scaled units, where 1 unit on the applied voltage scale corresponds to $30 \mathrm{~V}$.

\subsection{Materials and methods}

Argon plasma cleaner (Harrick Plasma, US) with pressure sensor was used for cleaning of ATcut 14.3 MHz quartz crystals (Laptech,_Canada) of working surface area $0.22 \mathrm{~cm}^{2}$. Palmsens 3 Potentiostat (Alvatek) was used in the electrochemical setup. 
Acetone, absolute ethanol, propan-2-ol, synthetic thiolated DNA (5'-3') AACTATGCAACCTACTACCTCTTTTT[ThiC3], 6-mercaptohexanol (MCH), synthetic complementary DNA (5'-3') AGAGGTAGTAGGTTGCATAGTT, potassium ferricyanide (III), potassium hexacyanoferrate (II), tris(2-carboxyethyl)phosphine hydrochloride solution (TCEP),

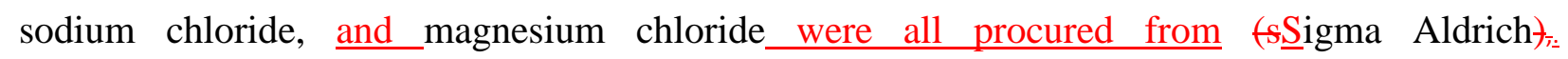
ethylenediaminetetracetic Ethylenediaminetetraacetic acid disodium salt dehydrate (EDTA), tris-EDTA buffer (TE Buffer), and $10 \mathrm{mM}$ phosphate buffer saline solution (PBS) (Fisher Scientific)-were procured from Fisher Scientific, UK, and used for surface cleaning and functionalisation as described below. $\mathrm{Ag} / \mathrm{AgCl}$ reference electrode was procured from Cambria Scientific.

Buffer Solutions: Two buffer solutions were used: hybridisation buffer and immobilisation buffer. Immobilisation buffer was $1 x T E$ buffer, $1 \mathrm{M} \mathrm{NaCl}, 50 \mathrm{mM} \mathrm{MgCl} 2$ and $10 \mathrm{mM}$ EDTA. Hybridisation buffer was $1 x T E, 1 \mathrm{M} \mathrm{NaCl}$ and $0.1 \mathrm{M} \mathrm{MgCl}_{2}$. After the immobilisation was complete, the same (hybridization) buffer was used in the baselining (in thiol and $\mathrm{MCH}$ ) and in the detection of ssDNA (hybridization) steps. So we can consider that the buffer made no contribution to the shifts in resonance frequency and third harmonic signal due to hybridization.

Crystal Cleaning: Crystals were incubated for $5 \mathrm{~min}$ in acetone with shaking followed by $5 \mathrm{~min}$ sonication in propan-2-ol. The crystal was then dried with nitrogen and cleaned in an argon plasma cleaner for $45 \mathrm{sec}$. The crystal was immersed in ethanol immediately after taking it out from the plasma cleaner. 
Thiolated DNA Reduction: Thiolated DNA was reduced with 0.5M TCEP in a 1:1 (v/v) ratio for

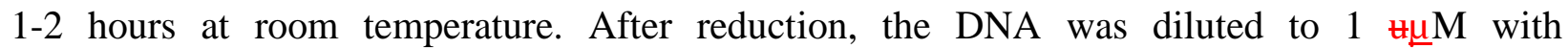
immobilisation buffer.

EQCM Assay: 1 씨 reduced thiolated DNA solution was placed on the crystal and a voltage of $0.08 \mathrm{~V}$ applied for 30 min. The crystal was then washed three times in PBS and incubated in 1 $\mathrm{mM}$ aqueous $\mathrm{MCH}$ for $60 \mathrm{~min}$ and washed three time is PBS. The analyte DNA was diluted to 1 muM in hybridization buffer the solution was then incubated on the crystal for 30 min and washed three time in PBS.

Cyclic Voltammetry and Impedance Spectroscopy: All measurements were conducted using a Palmsens 3 Potentiostat, platinum counter electrode, silver chloride reference electrode and the gold crystal working electrode. Cyclic voltammetry and impedance spectroscopy scans were taken in $5 \mathrm{mM}\left[\mathrm{Fe}(\mathrm{CN})_{6}\right]^{3-4-}$ in $10 \mathrm{mM}$ PBS. Cyclic voltammetry was conducted from $-0.1 \mathrm{~V}$ to $0.6 \mathrm{~V}$ at a scan rate of $0.03 \mathrm{~V} / \mathrm{s}$. Impedance spectroscopy was at a DC voltage of $0.0 \mathrm{~V}$ with open circuit potential and AC of $0.01 \mathrm{~V}$ from $50 \mathrm{MHz}$ to $0.1 \mathrm{~Hz}$.

ADT Scan: For an ADT scan, cyclic voltammetry was set from $-0.15 \mathrm{~V}$ to $0.6 \mathrm{~V}$ at a step of $0.0025 \mathrm{~V}$ and scan rate of $0.005 \mathrm{~V} / \mathrm{s}$. When the potential reached $-0.1 \mathrm{~V}, 0.1 \mathrm{~V}, 0.3 \mathrm{~V}$ and $0.5 \mathrm{~V}$ in the cyclic voltammetry, an $\mathrm{ADT}$ a frequency scan was taken with a scan time of $0.1 \mathrm{~s}$ and

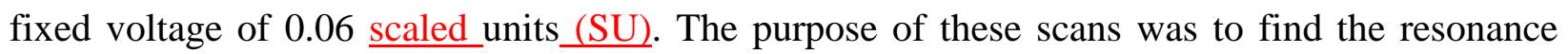
frequency. Subsequently amplitude ramps from 0 to $0.1 \mathrm{SU}(1 \mathrm{SU}=30 \mathrm{~V})$ during 0.1 s were applied at the same electrode potentials and fixed frequencies chosen near the fundamental resonance found from the previous frequency scans. The amplitude ramp is referred to as the ADT scan as the third Fourier harmonic signal (ADT signal) is evaluated from this scan. 
The electrode mounting, simultaneous connection to ADT and potentiostat were as are shown in Figure 2a, which is similar to our set-up published earlier [10]. Figure 2b shows steps of functionalization of the top gold electrode of EQCM.

\section{RESULTS}

\subsection{Electrochemical impedance spectroscopy}

Electrochemical impedance spectra after each step of electrode functionalization and hybridization stage are shown in Fig 3. The fitting was done to Randles circuit $R_{\mathrm{s}}+C_{\mathrm{dl}} \|\left(R_{\mathrm{ct}}+W\right)$, where $R_{\mathrm{s}}$ is solvent, $R_{\mathrm{ct}}$ charge transfer resistance respectively, $C_{\mathrm{dl}}$ is capacitance of double layer, $W$ is Warburg impedance. The resulting values of equivalent circuit parameters are shown in the Table 1 . The charge transfer resistance increases dramatically more than 10 times upon functionalization with thiolated ssDNA, but then falls 4.5 times when the spacer 6-mercaptohexanol is added. This means that effectively $\mathrm{MCH}$ acts as a shunting resistance, directing most of the electron transfer through it. This causes uncertainty in the interpretation of hybridization stage, since resistance changes on ssDNA, whereas the main current passes through $\mathrm{MCH}$.

\subsection{Anharmonic detection}

ADT signal (amplitude of third Fourier harmonic) during amplitude ramps were taken twice after each functionalization step at fixed potentials. Two frequency sweeps were also taken immediately after each set of amplitude scans to determine the fundamental resonance frequency. As an example, the ADT signals with ssDNA on the surface and after hybridization are shown in Fig 4. While the amplitude of voltage $a$ at fundamental frequency was linearly 
increased, the amplitude of induced third harmonic increased proportional to the third power of the applied voltage, according to Eq.7. Both curves in Fig 4 can be fitted with const $\times a^{3}$ function, where const stands for the pre-exponent for $a^{3}$. According to Eq.7, the const depends on the intrinsic anharmonic coefficient for quartz $\beta$ plus the term that depends on the number of flexibly-bound adsorbate $N S$ and properties of the bond, but not on attached mass, which remains immobile while the underneath surface moves in the laboratory reference frame. Therefore, different pre-exponents for the curves in Fig 4 reveal changes in the second term, which reflects the binding event.

Since all kinetic transients are cubic as a function of applied voltage, they differ only due to the pre-exponent. In other words, their final value depends on the pre-exponent, if the applied voltage ramp is the same. Figure 5a, which is the main experimental result of the work, shows

the final values of induced $3^{\text {rd }}$ harmonic for all stages of functionalization at 4 different electrode potentials. Figure 5b shows the shift of resonance frequency for the same conditions (stage of functionalization and potential) as Figure 5a. The reference point for frequency is that for the clean surface at each potential.

\section{DISCUSSION}

This work presents a study of thiolated ssDNA and $\mathrm{MCH}$ functionalization, and subsequent hybridization analysis using the nonlinear response of an EQCM. Traditional EIS does reflect changes at each step (Fig 3). However, since MCH has the lowest charge transfer $R_{\mathrm{ct}}$ resistance, it serves as a shunting element in the equivalent circuit (Table1). Since $R_{\mathrm{ct}}$ is in parallel with the charge transfer resistance for ssDNA and complementary ssDNA upon hybridization, it is difficult to make conclusions on ssDNA hybridization from the EIS data, although it may be 
useful in drawing equivalent circuits. Because of this limitation, a new technique, called anharmonic detection technique, or ADT, is probed here. ADT is based on the modification in the amplitude of the third Fourier harmonic of the nonlinear QCM when driven near its fundamental resonance frequency. The preceding theoretical analysis shows that any material attaching flexibly (not rigidly) to the QCM results in an anti-Sauerbrey response, i.e. binding results in increase in the fundamental resonance frequency. In our experiment, binding of ssDNA to thiolated DNA receptor functionalised on $14.3 \mathrm{MHz}$ QCM resulted in increase in resonance frequency of the QCM in the presence of electrode potential. However, similar binding resulted in decrease in resonance frequency in the absence of an electrode potential. Our hypothesis is that the presence of electrode potential together with the higher resonance frequency of the QCM used (14.3 MHz) contributed to the flexibility in the nature of attachment of the ssDNA to the QCM. The flexible attachment allowed the ssDNA to be almost 'pinned by inertia' in the laboratory reference frame, while the QCM oscillated underneath. This contributed to stretching of the ssDNA, and therefore, an elastic loading on the QCM. The positive (or anti-Sauerbrey) frequency shift caused by the dominant elastic loading could be related to the shift in the third Fourier harmonic amplitude using the Duffing equation. It may be noted that the elastic loading described above should not be confused with viscoelastic loading. Dissipation changes measured in our experiments was negligible. Hence, viscous damping from the ssDNA attachment could be considered insignificant, and potentially explained by the separation between the ssDNA and the QCM under the influence of a 'pull' due to the oscillation of the high-frequency (14.3 MHz)

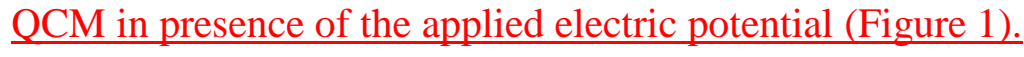

The nonlinear response calculated from the model Duffing equation results in changes in the fundamental frequency and the amplitude of third Fourier harmonic $x^{(3)}$ depending on the 
amplitude of excitation drive $a$ as $a^{2}$ (Eq. 5) and $a^{3}$ (Eq. 7) respectively. In the model of inertially-pinned mass attached by a flexible string, the pre-exponent depends on the nonlinear coefficient of the quartz itself $\beta$ and on the term $N k / l^{2}$ which accounts for the number of bound particles (per unit area) $N$, spring constant $k$, and length of the bond $l$.

Now we are in a position to interpret the steps of surface functionalization and hybridization as measured by the ADT method. The cubic curves in Fig 4 of induced third harmonics $x^{(3)}$ agrees with the model Eq.7, while their changing amplitude accounts for different $N k / l^{2}$ term defined by structure of adsorbed layer. With each functionalization step, the $x^{(3)}$ and the resonance frequency of the fundamental mode are growing, which agrees with Eq.5 and Eq.7 because they have the same pre-exponent. On a clean surface, the adsorbate related term is absent. So the $x^{(3)}$, and $f(=\omega / 2 \pi)$ are minimal. Upon addition of thiolated ssDNA, the aforementioned $\left(\mathrm{Nk} / \mathrm{l}^{2}\right)_{\mathrm{DNA}}$ term makes a positive contribution to the pre-exponent. This results in simultaneous growth of $f$ and $x^{(3)}$ as shown in Fig 5a,b. Further functionalization with MCH adds another $\left(\mathrm{Nk} / \mathrm{l}^{2}\right)_{\mathrm{MCH}}$ term related to this binding since it binds on free Au surface. Finally in the hybridization stage, the complementary ssDNA binds only to ssDNA already on the surface. This binding modifies the $\left(N k / l^{2}\right)_{\text {DNA }}$ term by increasing, possibly doubling, the spring constant $k$, since there is double DNA spiral in place of one. Therefore on each step there is positive addition to the pre-exponent, which manifests itself in grows of $f$ and $x^{(3)}$ on each step. This analysis is summarized in the Table 2.

In this simple approximation, the shift increase of $f(=\omega / 2 \pi)$ and $x^{(3)}$ in Fig 5 should be the same from the clean surface to thiol-ssDNA and in the $\mathrm{MCH}$ to hybridization step. This approximately holds for $x^{(3)}$ in Fig 5b, but not that well for the resonance frequency shifts in Figure 5a. Still, 
given the complexity of the system and simplifications made in analysis, the agreement seems to be satisfactory.

However, the following issues need to be discussed: the influence of electrode potential, the influence of solvent and validity of approximations made in iterative solutions of the Duffing equation. Although the nonlinear responses are different at 4 probed potentials, there is no clear trend evident. The model does not include the electrode potential either. The reason is that the bond length is much longer than double layer thickness, so one ean't cannot expect dramatic influence of the electrode potential in this particular system. Also, only lateral projection of external force influences movements in quartz, according to Eq.1, whereas the electric field is perpendicular to the surface.

The influence of solvent may be introduced by the loss term in Eq.1, as it was done in our earlier work [10]. However, here it was not necessary as the change in dissipation was negligible in the experiments as explained above. Also, in the model, the attached mass remains immobile in the laboratory frame of reference while the quartz oscillates. This motion induces motion of liquid within the decay length $(2 v / \omega)^{1 / 2}=10^{-5} \mathrm{~cm}$ or $100 \mathrm{~nm}$ for $14 \mathrm{MHz}$ oscillations in water, which is more than 10 times longer than ssDNA length $(\sim 8.99 \mathrm{~nm})$. Therefore, rigorous treatment should include influence of flow around the attached mass as well. At this point, one can argue that this will effectively lead to a heavier mass. However, in this work the mass is already considered to be infinitely heavy, so the mass does not appear in the equations.

The iterative solution of the resulting Duffing Eq.3 is valid in the first approximation. The nonlinear terms on the right hand of Eq.3 can be neglected and subsequently treated as perturbations. As shown in Eq.8, the resonance frequency shift due to binding of particles is 
linearly related to the corresponding (measured at the same driving amplitude $a$ ) pre-exponential factor shift, both normalized to the respective parameters before binding of particles. According to Eq. 5 and Eq. 7, this dependence must be a straight line passing through the origin with the slope $3 a^{2} \beta / 8 \omega_{0}^{2}$ for all stages of functionalization. Such a plot produced from the reported hybridisation experiment is shown in Fig 6. We do not see a straight line through origin, again due to oversimplification of the theoretical treatment. However, from the plot one can roughly estimate the value $3 a^{2} \beta / 8 \omega_{0}^{2} \sim 10^{-3}$, which is approximately the ratio between terms on the right and left hand side of Eq.3. It is important to note that this ratio depends on the driving amplitude as $a^{2}$. Therefore at a given amplitude $a=0.06$ scaled units, for which the plot is made, the iterative solution is valid, and should remain valid until amplitude at least 10 times larger, or $a=0.6$ units of scaled voltage. The maximum applied voltage in the current work was $a=0.1$ scaled units ( where 1 scaled unit $=30 \mathrm{~V}$ ).

\section{Selectivity of Detection}

Experiments were conducted with a random sequence using the same thiolated DNA receptor on the QCR. The shifts in third harmonic signal for different electrode potentials obtained with this random sequence were negligible (0-4\%) compared to that obtained from the complementary sequence (Figure 7a,c). However, the corresponding shifts in the resonance frequency, although smaller, were not insignificant (50-57\%) compared to that obtained from the complementary sequence (Figure 7b.d). Although the degree of non-complementarity of the sequences was not investigated as part of this work, and it is possible that the random sequence had some degree of affinity towards the receptor sequence, the specificity using the third harmonic signal is comparatively much better. Improved specificity in transduction using third harmonic shift signal agrees with that observed in our previous work $[7,8,22]$. 


\section{CONCLUSION}

Anharmonic acoustic detection method (ADT) is applied to the monitoring of EQCM functionalization with thiolated ssDNA, MCH and hybridization with complementary ssDNA. The results can be interpreted using a simple nonlinear model for oscillation of quartz crystal resonator coupled with a flexibly attached mass. Both the fundamental resonance frequency and the amplitude of nonlinearly generated third Fourier harmonic increase with the formation of adsorbed layers. Hybridization event is detected due to increased strengthening of the bond, and not by mass change.

\section{AUTHOR INFORMATION}

\section{Corresponding Authors}

* igor.efimov@ntu.ac.uk,*s.ghosh2@lboro.ac.uk

\section{Author Contributions}

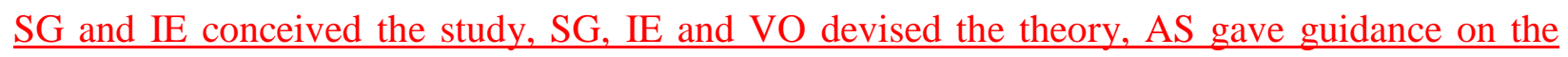
assay, VO developed the instrumentation with financial support from DK, SS designed the microfluidic flowcell, performed the experiments, and analysed the results, SS, IE and SG wrote the paper, and all authors discussed the results and reviewed the paper. The manuscript was written through contributions from all authors. All authors have given approval to the final version of the manuscript. 


\section{Funding Sources}

The authors sincerely acknowledge the funding support from the EU projects RAPP-ID (FP7-JTI 115153) and Norosensor (FP7-NMP 604244), EPSRC Bridging the Gap in Antimicrobial Resistance grant (EP/M027341/1) and PhD studentship support for SS from EPSRC Doctoral Training Centre (EP/F500491/1). 


\section{REFERENCES}

1. Zhu, Q., G. Liu, and M. Kai, DNA Aptamers in the Diagnosis and Treatment of Human Diseases. Molecules, 2015. 20(12): p. 20979-97.

2. Rand, K., H. Houck, and R. Lawrence, Real-time polymerase chain reaction detection of herpes simplex virus in cerebrospinal fluid and cost savings from earlier hospital discharge. $\mathrm{J}$ Mol Diagn, 2005. 7(4): p. 511-6.

3. Luo, X. and J.J. Davis, Electrical biosensors and the label free detection of protein disease biomarkers. Chem Soc Rev, 2013. 42(13): p. 5944-62.

4. Park, J.Y. and S.M. Park, DNA hybridization sensors based on electrochemical impedance spectroscopy as a detection tool. Sensors (Basel), 2009. 9(12): p. 9513-32.

5. $\quad$ Caruso, F., et al., Quartz crystal microbalance study of DNA immobilization and hybridization for nucleic Acid sensor development. Anal Chem, 1997. 69(11): p. 2043-9.

6. D. Johannsmann, The Quartz Crystal Microbalance in Soft Matter Research: Fundamentals and Modeling. Springer, 2014.

7. Ghosh, S.K., V.P. Ostanin, and A.A. Seshia, Anharmonic Surface Interactions for Biomolecular Screening and Characterization. Analytical Chemistry, 2011. 83(2): p. 549-554.

8. Ghosh, S.K., V.P. Ostanin, and A.A. Seshia, Anharmonic Interaction Signals for Acoustic Detection of Analyte. Analytical Chemistry, 2010. 82(9): p. 3929-3935.

9. Ghosh, S.K., V.P. Ostanin, and A.A. Seshia, Studying adsorbent dynamics on a quartz crystal resonator using its nonlinear electrical response. Sensors and Actuators B-Chemical, 2013. 176: p. 577-584.

10. Khobragade, S., et al., Detection of flexibly bound adsorbate using the nonlinear response of quartz crystal resonator driven at high oscillation amplitude. Electrochimica Acta, 2017. 252: p. 424-429

11. L. D. Landau, E. M. Lifshitz, Mechanics Course of Theoretical Physics Mechanics, Pergamon Press, 1969. Chapter 5, p. 112-122.s.

12. Kovacic, I., et al., Jacobi elliptic functions: A review of nonlinear oscillatory application problems. Journal of Sound and Vibration, 2016. 380: p. 1-36.

13. Berglin, M., A. Olsson, and H. Elwing, The interaction between model biomaterial coatings and nylon microparticles as measured with a quartz crystal microbalance with dissipation monitoring. Macromol Biosci, 2008. 8(5): p. 410-6.

14. Olofsson, A.C., M. Hermansson, and H. Elwing, Use of a quartz crystal microbalance to investigate the antiadhesive potential of N-acetyl-L-cysteine. Appl Environ Microbiol, 2005. 71(5): p. 2705-12.

15. D'Amour, J.N., et al., Capillary aging of the contacts between glass spheres and a quartz resonator surface. Phys Rev Lett, 2006. 96(5): p. 058301.

16. Dybwad, G.L., A SENSITIVE NEW METHOD FOR THE DETERMINATION OF ADHESIVE BONDING BETWEEN A PARTICLE AND A SUBSTRATE. Journal of Applied Physics, 1985. 58(7): p. 2789-2790.

17. Pomorska, A., et al., Positive Frequency Shifts Observed Upon Adsorbing Micron-Sized Solid Objects to a Quartz Crystal Microbalance from the Liquid Phase. Analytical Chemistry, 2010. 82(6): p. 2237-2242.

18. Olsson, A.L.J., et al., Probing Colloid-Substratum Contact Stiffness by Acoustic Sensing in a Liquid Phase. Analytical Chemistry, 2012. 84(10): p. 4504-4512.

19. Fourati, N., et al., Surface Acoustic Waves Sensor for DNA-Biosensor Development. Sensor Letters, 2009. 7(5): p. 847-850. 
20. Uludag, Y., et al., Direct acoustic profiling of DNA hybridisation using HSV type 1 viral sequences. Analyst, 2008. 133(1): p. 52-7.

21. Gabl, R., et al., First results on label-free detection of DNA and protein molecules using a novel integrated sensor technology based on gravimetric detection principles. Biosensors and Bioelectronics, 2004. 19(6): p. 615-620

22. Ghosh, S.K., et al., Probing biomolecular interaction forces using an anharmonic acoustic technique for selective detection of bacterial spores. Biosensors and Bioelectronics, 2011. 29(1): p. $145-150$.

\section{FIGURE CAPTIONS}

Figure 1. Schematic presentation of the model in the frame of moving quartz. Mass $m_{\mathrm{s}}$ is attached to the quartz with the spring of length $l$ and spring constant $k$. Mass is heavy, so in the laboratory frame it is resting, while quartz oscillating. In the frame of quartz the mass is moving parallel to the surface. Only projection of the force $F_{\mathrm{x}}$ on the quartz surface affects shear thickness mode of oscillations. This leads to additional quatric $x^{4}$ potential for the quartz oscillations even if the spring itself is harmonic.

Figure 2. a) Electrochemical flow cell and mount of the quartz crystal with connection to ADT and potentiostat. b) Schematic of gold EQCM functionalization. I) cleaned gold electrode, II) Addition of $1 \mu \mathrm{M}$ thiolated ssDNA after reduction in TCEP for 30 mins with added $0.08 \mathrm{~V}$, III) Back functionalization with $1 \mathrm{mM} \mathrm{MCH}$ for hour, IV) Incubation with complimentary DNA for 30 mins

Figure 3. Cyclic voltammetry (a) and Nyquist plot and Randles circuit (b) electrode, after functionalization of $1 \mu \mathrm{M}$ thiolated ssDNA, $-1 \mathrm{mM} \mathrm{MCH}$ and hybridization of the $1 \mu \mathrm{M}$ complementary sequence conducted in $1: 1(\mathrm{v} / \mathrm{v}) 5 \mathrm{mM} \mathrm{Fe} \mathrm{II}^{\mathrm{II}}$ and $5 \mathrm{mM} \mathrm{Fe}^{\mathrm{III}}$ in PBS. 
Figure 4. ADT third harmonic increase with driving voltage for a thiolated DNA surface and with attached complementary target sequence after hybridization

Figure 5. (a) $\underline{x^{(3)}}$ Fundamental resonance frequency shift and (b) fundamental resonance frequency shift $x^{(3)}$ taken respectively from e-QCM-amplitude and frequency scans of the gold EQCM crystal surface after addition of $1 \mu \mathrm{M}$ reduced thiolated ssDNA-, $1 \mathrm{mM}$ MCH and the complementary sequence. (ab) was normalized to the clean surface. Potentials are indicated. The error bars are presented as $+/$ - half the range for duplicate measurements. The error bars are too $\underline{\text { small to be seen for most measurements. }}$

Figure 6. Relative frequency shift as a function of relative amplitude of $3^{\text {rd }}$ harmonic measured at the same actuation amplitude $\mathrm{a}=0.06$ for various functionalization stages at several indicated potentials

Figure 7. Selectivity of detection using a random sequence. The third harmonic signal (a) and the resonance frequency shift with respect to bare ('clean') QCM (b) after the final stage of thiol functionalization (with $\mathrm{MCH}$ ) and after hybridization with a random (non-complementary) sequence at various electrode potentials. The comparision of shifts in third harmonic signal (c) and of resonance frequency (d) due to hybridization with complementary and noncomplementary sequence at various electrode potentials are presented. The error bars are presented as + - - half the range for duplicate measurements.

FIGURES 


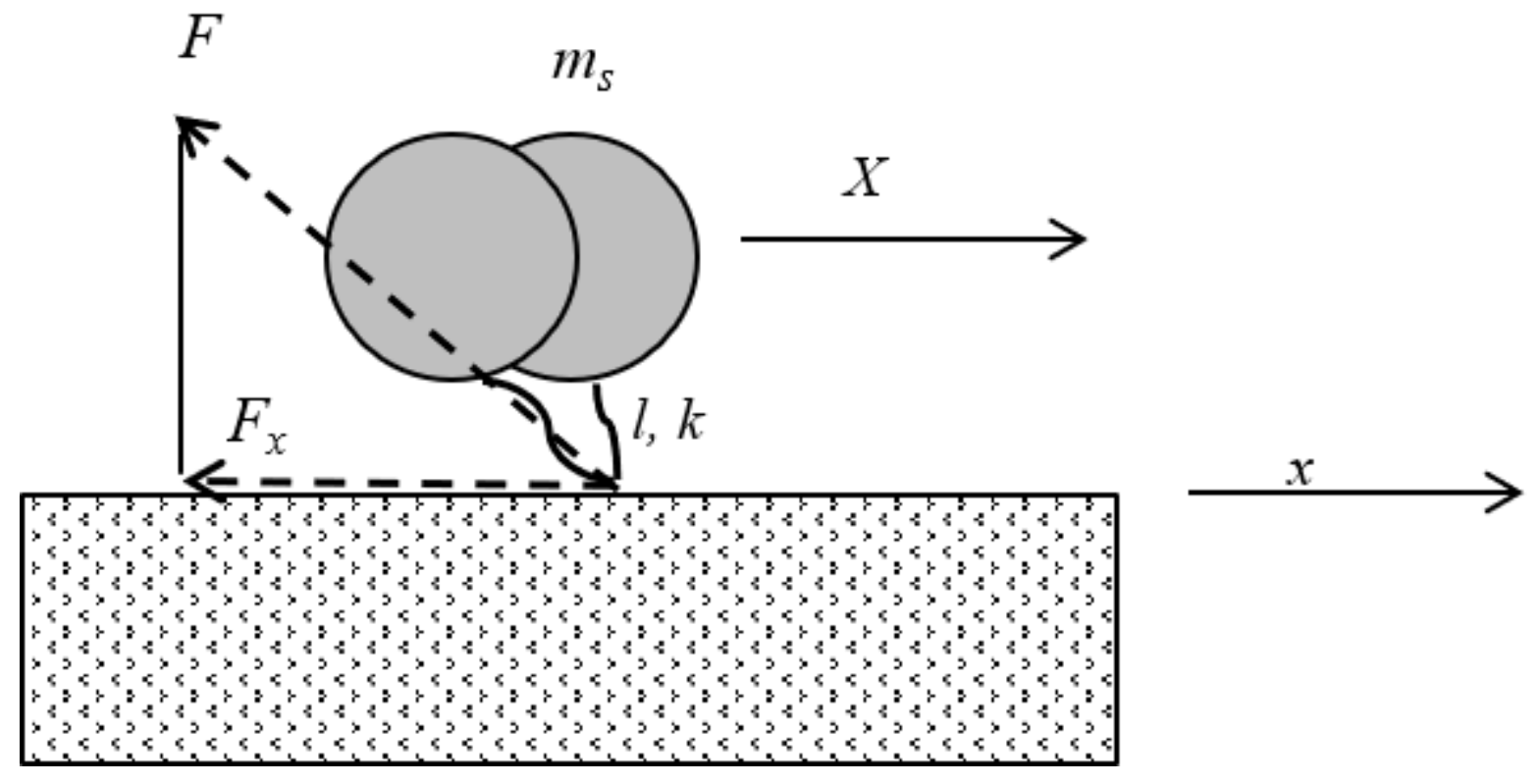

Figure 1 
a
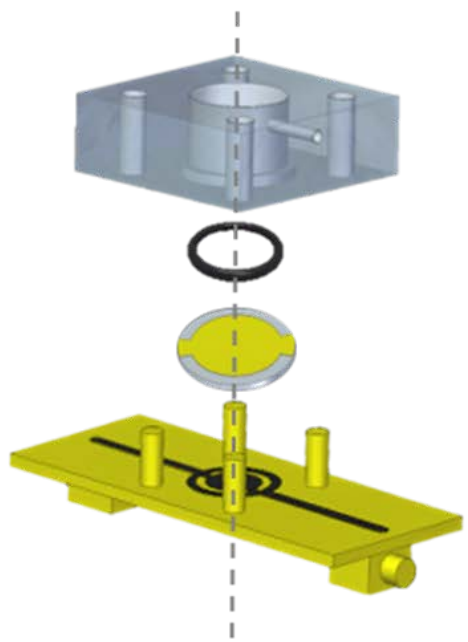

b

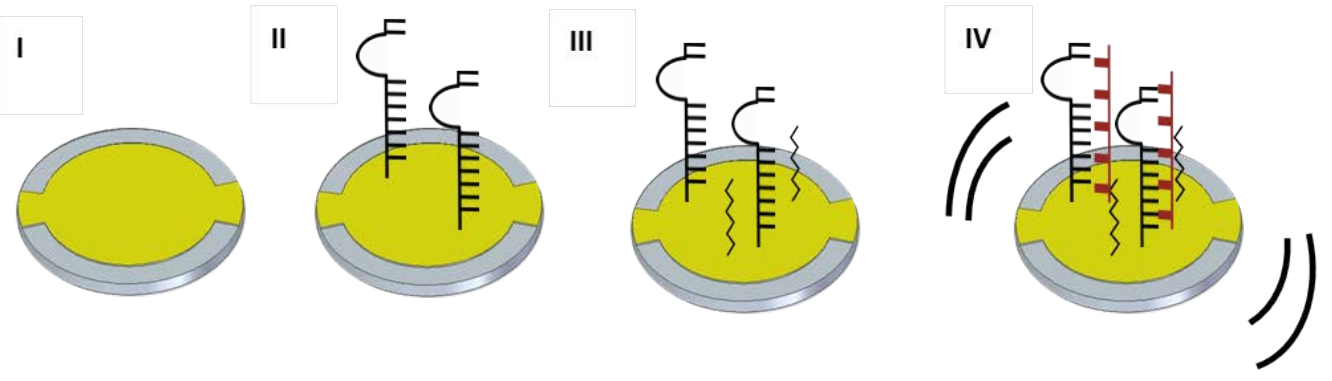

Figure 2 

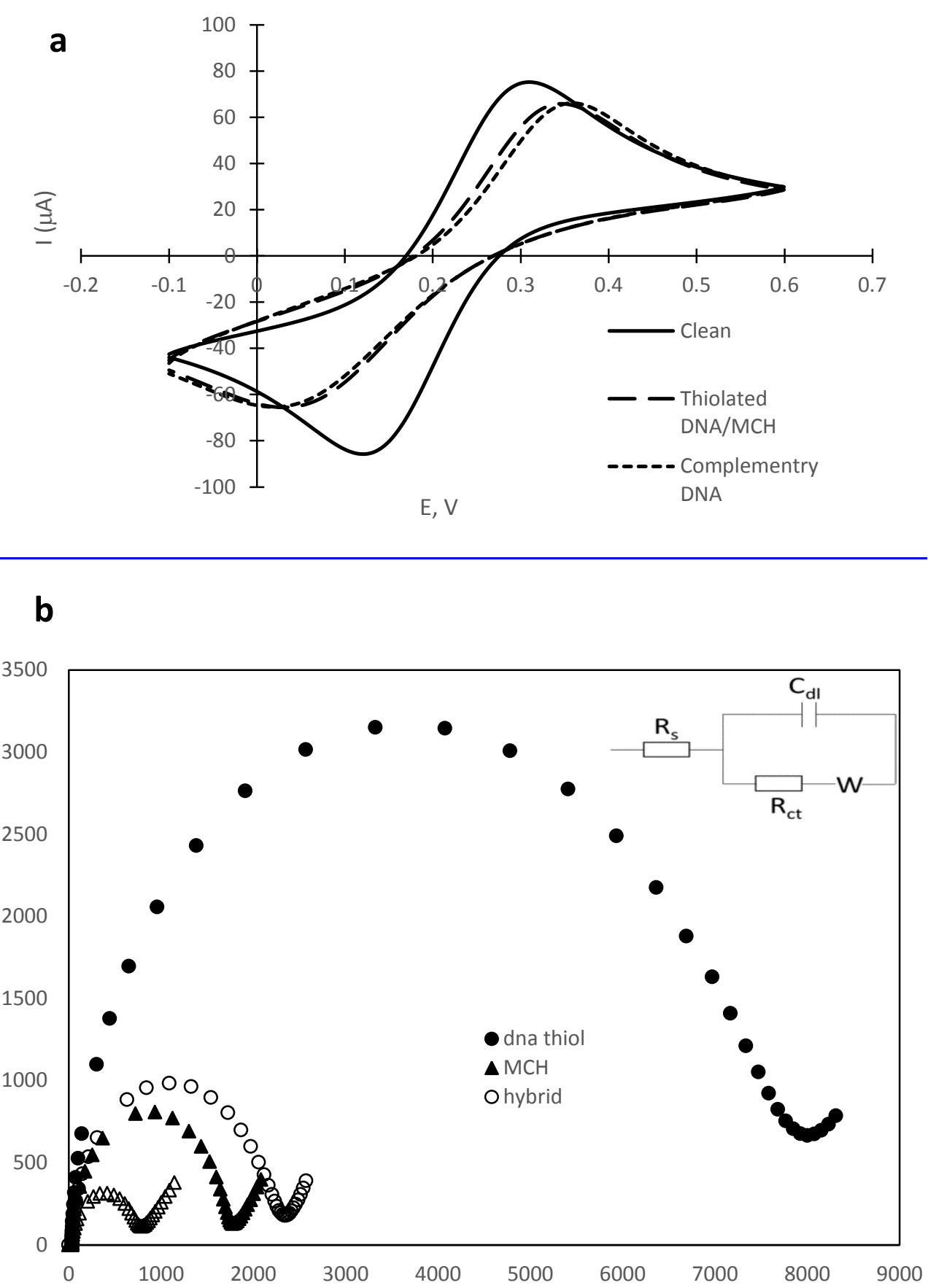

Figure 3 


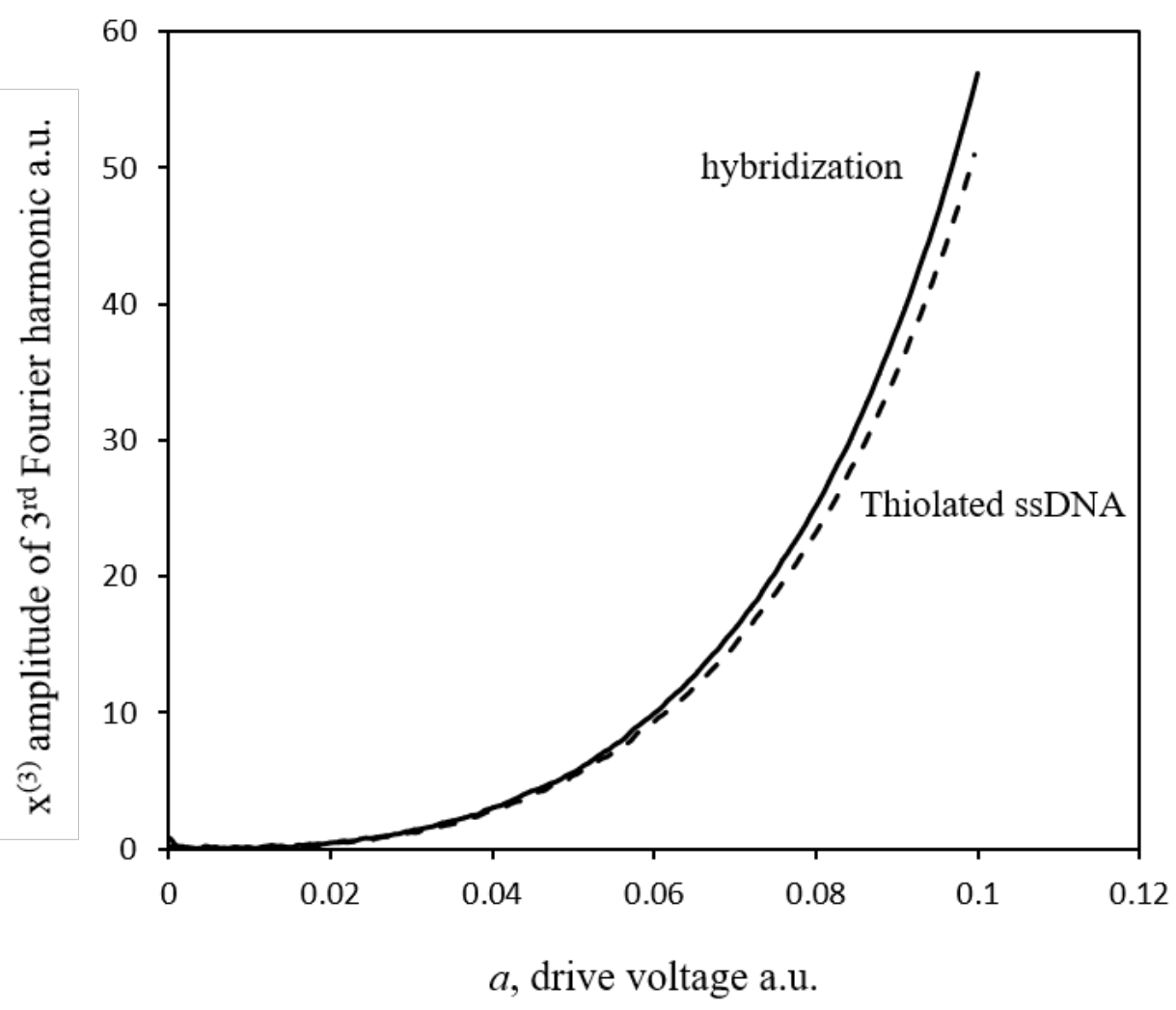

Figure 4 


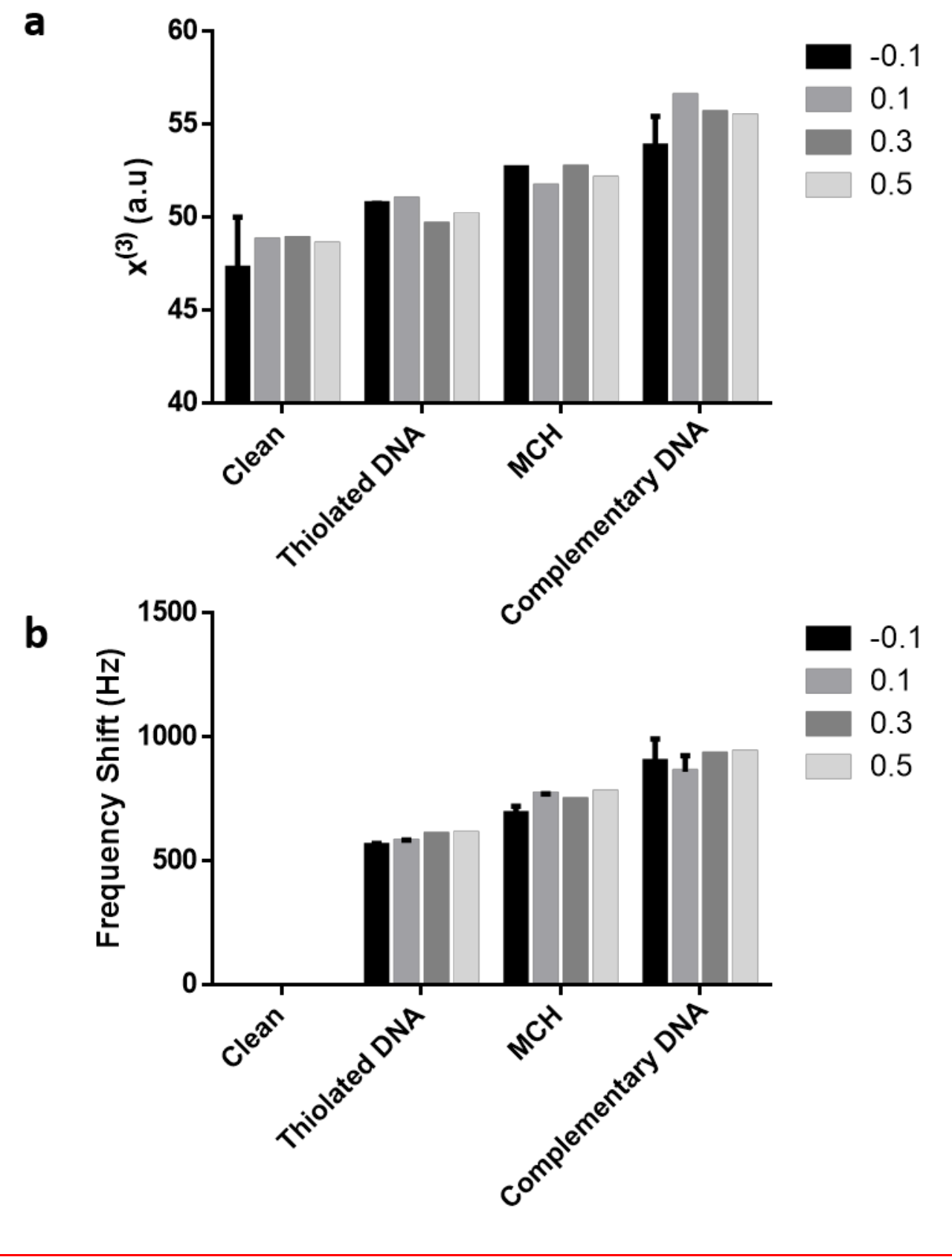

Figure 5 


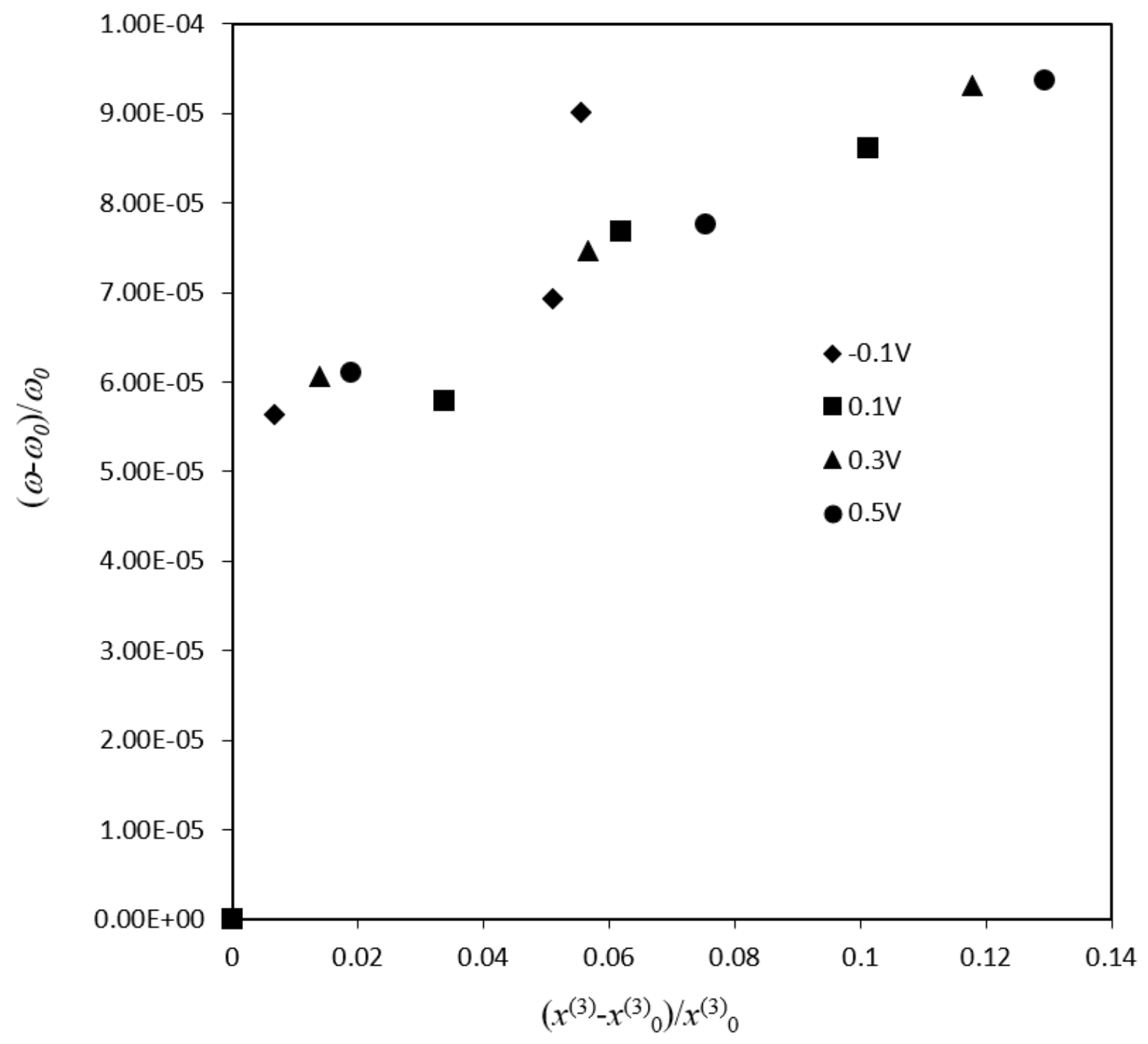

Figure 6 
a

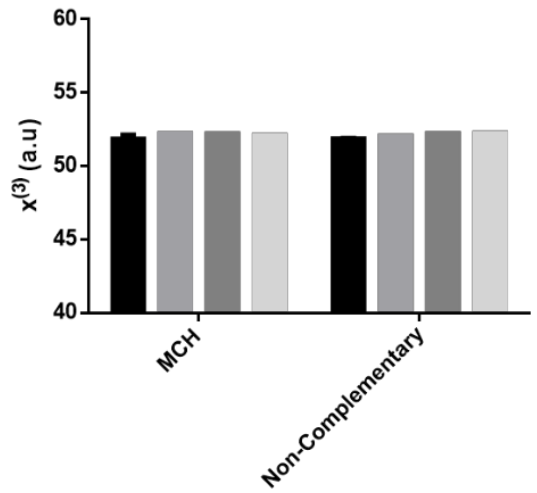

b

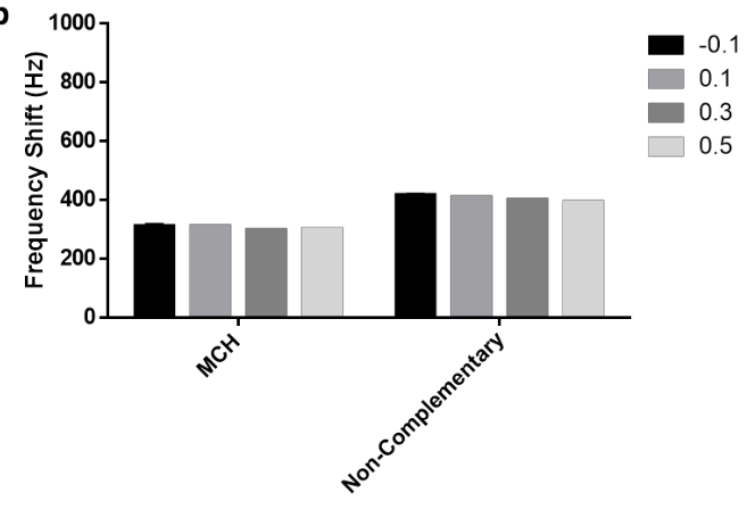

c

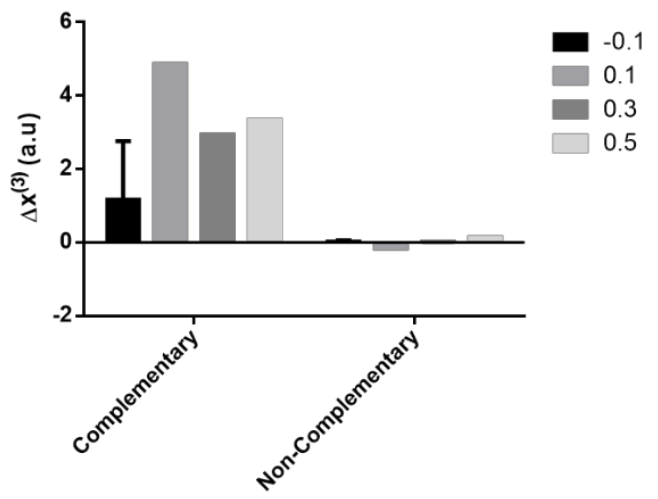

d

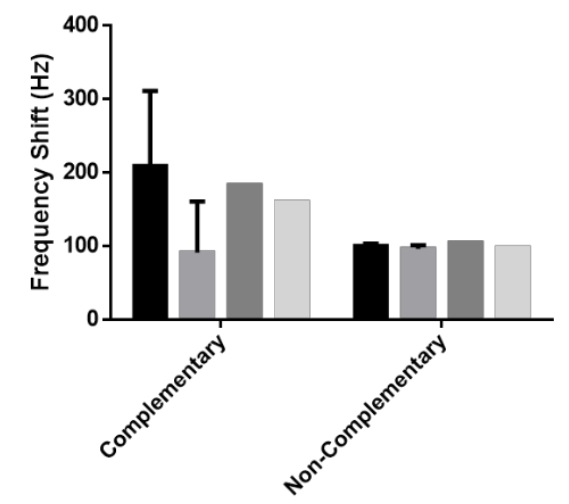

- 0.1

0.1

0.3

$\square 0.5$

Figure 7 


\section{TABLES}

\begin{tabular}{|l|c|c|c|c|}
\hline \multicolumn{1}{|c|}{} & & & & \\
interface & $R_{s}, \mathrm{Ohm}$ & $R_{c t}, \mathrm{Ohm}$ & $C_{d l}, \mu \mathrm{F}$ & W, Ohm \\
\hline Clean surface & 35 & 681 & 20 & 137 \\
\hline Thiol DNA & 37 & 7024 & 12 & 131 \\
\hline MCH & 36 & 1664 & 5.3 & 128 \\
\hline hybrid & 36 & 2155 & 6.1 & 140 \\
\hline
\end{tabular}

Table 1 Randles equivalent circuit parameters for impedance spectra shown in Fig 3.

\begin{tabular}{|l|l|}
\hline Functionalization step & Pre-exponent in parentheses Eq. 6,7 \\
\hline Clean surface & $\beta$ \\
\hline Thiol-ssDNA & $\beta+\left(S / 2 M_{\mathrm{q}}\right)\left(N k / l^{2}\right)^{-2}$ DNA \\
\hline MCH & $\beta+\left(S / 2 M_{\mathrm{q}}\right)\left(\left(N k / l^{2}\right)_{\mathrm{DNA}}+\left(N k / l^{2}\right)_{\mathrm{MCH}}\right)$ \\
\hline Hybridization & $\beta+\left(S / 2 M_{\mathrm{q}}\right)\left(2\left(N k / l^{2}\right)_{\mathrm{DNA}}+\left(N k / l^{2}\right)_{\mathrm{MCH}}\right)$ \\
\hline
\end{tabular}

Table 2. Pre-exponent in the model equations 6 and 7 for different functionalization stages. Subindex DNA or $\mathrm{MCH}$ means that all parameters in parentheses $(N, k, l)$ are relevant for corresponding adsorbate. 